\title{
Formación de competencias: tendencias y desafíos en el siglo XXI
}

\section{José Lino Contreras V.*}

\section{Resumen}

Las competencias han adquirido una relevancia especial en diversos sectores de la actividad social en los últimos años, en particular en el ámbito laboral y educativo. Frente a los nuevos desafíos que plantean a las personas y a las organizaciones los cambios profundos en la sociedad, derivados de la globalización de la economía, los avances en ciencia y tecnología, y la complejidad y alcance de los problemas, entre otros, los países están respondiendo con reformas en sus sistemas educativos que incluyen el enfoque de competencias como un factor orientador importante.

\section{Palabras clave}

Formación de competencias, cambios y nuevas demandas, formación profesional, curriculum y planes de estudio, evaluación.

\begin{abstract}
The skills have acquired a special significance in various sectors of social activity in recent years, particularly in the workplace and in education. Facing the new challenges posed to the people and organizations the profound changes in society, result of the economic globalization, science and technology advances and the complexity and scope of the problems, among others, the countries are responding with reforns in their educational systems that include the competence approach as an important guiding factor.
\end{abstract}

\section{Key words}

skills training, changes and new demands, training, curriculum, assessment.

Forma sugerida de citar:

Contreras, José Lino. 2011. "Formación de competencias: tendencias y desafíos en el siglo XXI”. Universitas 15. Julio/Diciembre. Pp. 109-138. 


\section{Introducción}

El tema de las competencias ha adquirido una relevancia especial en diversos sectores de la actividad social en las últimas décadas, particularmente en los ámbitos laboral y educacional. Numerosas iniciativas de alcance nacional y también internacional, en todo el mundo, están cambiando la forma cómo se definen los puestos de trabajo, cómo se gestionan las capacidades laborales del personal y cómo se forman los profesionales, teniendo las competencias como eje orientador y articulador. Algunos ejemplos de ello son los trabajos sobre competencias laborales de la Organización Internacional del Trabajo (OIT), las políticas nacionales para la certificación y acreditación de competencias en varios países, como la Ley de Acreditación de Competencias Laborales en Chile, el proyecto de certificación y normas profesionales de Canadá, el National Skills Standards Board en USA, el Learning and Skills Council en Inglaterra y el Plan Nacional de Calificación en Brasil. Por otro lado, se están desarrollando proyectos internacionales de gran alcance, orientados a reformar y articular los sistemas de educación superior de los países participantes, entre los cuales se destacan los proyectos Tuning de la Comunidad europea y su análogo en América Latina. A nivel de países se están impulsando reformas que fomentan el enfoque de competencias en educación a través de iniciativas legislativas como en España, o vía programas de mejoramiento de la calidad de la educación superior como Mecesup en Chile y PMQESU en Brasil. También son numerosas las instituciones de educación superior que han actualizado sus modelos educativos basados en el enfoque de competencias, como el Instituto Tecnológico de Estudios Superiores de Monterrey en México, la Universidad de Deusto en España, entre muchísimas otras más en el mundo.

Las acciones mencionadas en el párrafo anterior dan cuenta del enorme movimiento que ha suscitado el tema de las competencias y de la formación de competencias en todo mundo. Si bien este interés se podría visualizar como un fenómeno propio de las sociedades modernas, el interés en las competencias responde a una necesidad ancestral del hombre, pues desde siempre las comunidades humanas han procurado desarrollar y transferir a las nuevas generaciones los saberes que dan respuesta apropiada a los desafíos que plantea la naturaleza y el mundo social. 
Con relación al especial interés por las competencias en las últimas décadas, su impulso se debió, principalmente, a iniciativas de los gobiernos de los EE.UU. y de Inglaterra, quienes en los años sesenta debieron mejorar la competitividad de sus países frente a la participación de nuevos protagonismos en la economía mundial. En un estudio encargado por el gobierno de los EE.UU. en 1973, McClelland concluyó que eran las competencias, más que los conocimientos, las que hacían la diferencia entre un desempeño profesional destacado frente a uno normal. A medida que avanzaron los cambios en diversos ámbitos del mundo social y tecnológico, el enfoque de competencias fue sumando adherentes en diversos países y organizaciones del mundo, encontrándose hoy presente en las organizaciones y en los sistemas de educación como un enfoque más adecuado para formar y gestionar el capital humano. Una mirada al contexto socioeconómico general al cual deben responder las personas, las organizaciones y los países en las sociedades modernas, ayudará a comprender mejor el interés puesto en las competencias.

En lo que sigue del documento, se presenta un breve análisis sobre el tema de las competencias y sus principales consecuencias en las personas y las organizaciones, con una particular mirada a los desafíos que plantea a la educación superior.

\section{Cambios en el mundo contemporáneo}

En las últimas décadas, la humanidad ha experimentado cambios sin precedentes en diversos ámbitos del mundo social y tecnológico, generando nuevos desafíos a las personas y a las organizaciones, quienes deben desenvolverse en escenarios más complejos, dinámicos e inciertos. Muchos estudios de alcance internacional se han referido a los retos que la humanidad deberá enfrentar en el futuro próximo. La Comisión Internacional sobre la educación para el siglo XXI, de la UNESCO, menciona que los problemas de la humanidad en el siglo XXI tendrán en su centro las siguientes tensiones (Delors, 1996):

- Entre lo mundial y lo local.

- Entre lo universal y lo singular.

- Entre la tradición y la modernidad.

- Entre el largo plazo y el corto plazo. 
- Entre la indispensable competencia y la preocupación por la igualdad de oportunidades.

- Entre el desarrollo de los conocimientos y las capacidades de asimilación del ser humano.

- Entre lo espiritual y lo material.

Muchos y diversos factores se combinan para perfilar las características dominantes de las sociedades modernas. A continuación se presenta una lista que resume los más relevantes:

- La globalización, en particular de la economía, que genera nuevas condiciones para los mercados, los sistemas productivos y las finanzas, así como también para la educación, las condiciones laborales, entre otros.

- Los avances en ciencia y tecnología, con dinamismos sin precedentes en la generación de nuevos conocimientos, a niveles imposibles de alcanzar por las personas y las organizaciones, y la creación y evolución acelerada de productos tecnológicos sofisticados.

- Las ideología neoliberal en políticas gubernamentales, que reducen la participación del Estado en la actividad social y económica, dejando en manos del sector privado, bajo una lógica de mercado, la provisión de servicios básicos tales como la energía, el agua potable, la salud, la educación, la vivienda, la generación de empleo, etcétera, generando nuevos escenarios sociales para las personas y las organizaciones.

- Problemas más complejos, multidimensionales e inéditos, de alcance internacional, donde se destacan la necesidad de suministros básicos para la vida tales como el agua potable, la energía y el alimento. Además el alcance de nuevas enfermedades, y problemas medio ambientales como el calentamiento global, la contaminación, la destrucción de la biósfera.

- Condiciones de mayor dinamismo, inestabilidad e incertidumbre en el sector productivo y el mercado laboral, entre otros. Ambientes de trabajo multiculturales y multidisciplinares, con participación de realidades económicas, culturales, legislativas, sociales y tecnológicas diversas, y aumento de la migración.

En los nuevos escenarios sociales y tecnológicos las personas deberán desarrollar nuevas competencias, particularmente quienes ejercerán profesionalmente. 


\section{Nuevas demandas a las personas}

Una mirada al mundo laboral y productivo contemporáneo hace pensar que las personas, como profesionales, deberán prepararse, entre otros, para:

...trabajar con otras personas, en contextos multidisciplinarios y multiculturales, ya sea en forma directa o a distancia, en escenarios nacionales o internacionales... para enfrentar desafíos complejos, inéditos, abiertos, ... con consideración de respeto a las personas y su cultura, el cuidado del medio ambiente, la legislación vigente...

En el campo de la ingeniería, Jack Lohmann, presidente de la American Society of Engineering Education, presentó una lista de cualidades que los "ingenieros globales" deberán poseer. Estas son válidas para cualquier profesional y se resumen en el siguiente párrafo (Lohmann, J., 2008. sitio web.):

El profesional del mundo globalizado debe ser un profesional sensitivo a las culturas, con conciencia social y astucia política, poseedor de un conocimiento amplio, con capacidad y disposición para aprender durante toda la vida, participante de equipos multidisciplinarios, un comunicador efectivo, con capacidad para hablar en lenguas extranjeras, sólidamente ético, innovador, emprendedor, flexible, con capacidad y disposición a movilizarse, ...

James Plummer, decano de la facultad de ingeniería de la universidad de Stanford, expuso recientemente sobre la necesidad de reformas en educación en ingeniería, mencionando algunas características que debieran formarse en los profesionales:

Las escuelas de ingeniería deben formar personas con conocimiento profundo de sus disciplinas y al mismo tiempo con conocimientos amplios en áreas más allá de las matemáticas y las ciencias básicas. Deben enseñar a los estudiantes cómo innovar, ser creativos y emprendedores; cómo trabajar bien como integrante de diversos equipos y desarrollar mejores habilidades de comunicación.

Lo anterior pone de manifiesto el nuevo escenario de trabajo que espera a los profesionales y las nuevas cualidades que éstos deberán poseer. Además de 
los conocimientos propios asociados a la disciplina, los profesionales deberán desarrollar otras competencias para desempeñarse en el mundo moderno.

\section{La noción de competencias}

El diccionario de la lengua española, Espasa-Calpe S.A., define competencia como: "Aptitud o capacidad para llevar a cabo una tarea". En términos simples, entonces, hablar de competencias es referirse a las capacidades que las personas ponen en acción al momento de realizar una tarea. Estas capacidades incluyen elementos tan diversos como conocimientos teóricos y prácticos, habilidades técnicas, de análisis, de gestión, de interacción social, capacidades motrices, relaciones sociales, entre muchas otras. Por otro lado, una persona es considerada competente para realizar una tarea cuando es capaz de realizarla cumpliendo los criterios de satisfacción establecidos para la tarea. Estas nociones simples sobre las competencias y el ser competente, no son tan simples de manejar cuando se trata de formar, evaluar o certificar competencias. Esto lo demuestra la gran cantidad de nociones, modelos y visiones desarrollados en torno al tema de las competencias que se ha producido en las últimas décadas, lo que a su vez ha generado grandes reacciones, posturas y dificultades con relación al tema. Tomemos un ejemplo simple para ilustrar su complejidad.

El éxito o fracaso en la realización de una tarea dependerá, por un lado, de las características propias de la tarea y de los criterios que se establezcan para su realización y, por otro lado, de las capacidades que tenga la persona que la realiza para cumplir con ellas. A modo de ejemplo, consideremos un despachador de pizzas cuya tarea es llevar una pizza desde la pizzería al domicilio del cliente, y debe emplear para ello un tiempo no mayor a 15 minutos. Una realización exitosa de la tarea se da cuando el despachador llega con la pizza al domicilio del cliente en un tiempo igual o menor que 15 minutos desde que dejó la pizzería. Si esta condición se cumple, entonces se logró el objetivo de la tarea de llevar la pizza al domicilio del cliente, y el criterio asociado a la realización de la tarea, el hacerlo en un tiempo no mayor a 15 minutos. Para esta tarea se podría pensar que un despachador competente es aquél que llega con la pizza al domicilio del cliente dentro del tiempo estipulado. ¿Qué pasa si el despachador toma más de 15 minutos para llegar al domicilio del cliente? ¿Qué 
pasa si llega en menos de 15 minutos, pero con la pizza en malas condiciones (fría, o desarmada, por ejemplo)? ¿Qué pasa si por alguna razón al cliente le parece mal como se expresa o como está vestido el despachador de pizzas? Estas, y otras situaciones, serían motivo para pensar que el despachador no cumplió bien con la tarea encomendada, en consecuencia, no es competente para ella. ¿Es esto tan claro? ¿Cuántos casos de no cumplimiento de las condiciones de la tarea son necesarios para hacer conclusiones sobre el nivel de competencia del despachador? ¿Qué pasa si "la mayor de las veces" cumple con "bien" con la tarea? ¿Cuánto sería "la mayor de las veces" y qué factores inciden en cumplir "bien" con la tarea? Todas estas y muchas otras interrogantes dicen de la relación de complejidad que hay detrás del tema de las competencias, particularmente para su evaluación.

A continuación se presentan algunas nociones de competencias presentes en los ámbitos laborales y formativos.

\section{Definiciones de competencia}

Sobre las competencias ha habido una cantidad enorme de estudios, conferencias y congresos realizados durante los últimos años. Baste decir que en el año 2004, una búsqueda en Internet de la palabra "competencia" arrojaba 1'040.000 documentos. Esta cantidad subió a 23'300.000 el año 2006 y a 30'400.000 el 2008. Un tema que ha despertado bastante controversia con relación a las competencias es el hecho que hay una cantidad enorme de definiciones y conceptualizaciones respecto al tema. Para ilustrar esta situación, se presentan a continuación algunas de las definiciones de competencia que han expresado estudiosos y organizaciones internacionales:

Las capacidades para transferir destrezas y conocimientos a nuevas situaciones dentro de un área de ocupación laboral; abarca la organización y la planificación del trabajo, la innovación y la capacidad para abordar actividades no rutinarias; incluye las cualidades de eficacia personal que se necesitan en el puesto de trabajo para relacionarse con los compañeros, los directivos y los clientes (Graham, S. A. et al., 1991: 285-293., citado en <http://www.nwrel.org/ $\mathrm{scpd} / \mathrm{sirs} / 8 / \mathrm{c} 015 . \mathrm{html}>$ ) 
Conjunto de saberes puestos en juego por los trabajadores para resolver situaciones concretas de trabajo, configurando una disociación entre competencia y acción, con una exigencia de determinado conocimiento que oriente esta acción (Gallart, M. A. y C. Jacinto, s/f).

Una estructura basada en recursos personales (conocimientos, habilidades, cualidades o aptitudes) y recursos ambientales (relaciones, documentos, información) que se movilizan para lograr un desempeño (Le Boterf, 2001).

El conjunto de saberes (saber, saber hacer, saber estar y saber ser conocimientos, procedimientos y actitudes) combinados, coordinados e integrados en el ejercicio profesional" (Tejada, J. Revista Herramientas, 57, s/f).

Característica subyacente en el individuo que está causalmente relacionada con un estándar de efectividad y/o a una performance superior en un trabajo o situación (Spencer, L.M. y S.M. Spencer, s/f).

La capacidad para actuar con eficiencia, eficacia y satisfacción sobre algún aspecto de la realidad personal, social, natural o simbólica (Pinto, L. RevistaTarea, 43, s/f).

Una capacidad de movilizar diversos recursos cognitivos para enfrentar un tipo de situaciones (Perrenoud, 2000).

Competencia es transformar el conocimiento en acción. Otra definición que propondríamos: "competencia es la combinación integrada de un saber, un saber hacer, un saber ser y un saber ser con los demás; que se ponen en acción para un desempeño adecuado en un contexto dado" (Irigoin, M. y F. Vargas, 2002).

un saber actuar complejo que se apoya en la movilización y combinación eficaces de una variedad de recursos internos y externos al interior de una familia de situaciones (Tardif, J. L, s/f).

La capacidad de responder a demandas complejas y llevar a cabo tareas diversas de forma adecuada. Supone una combinación de habilidades prácticas, conocimientos, motivación, valores éticos, actitudes, emociones y otros componentes sociales y de comportamiento que se movilizan conjuntamente para lograr una acción eficaz (Rychen y Hersh, s/f).

Capacidad de articular y movilizar condiciones intelectuales y emocionales en términos de conocimientos, habilidades, actitudes y prácticas, necesarias para el desempeño de una determinada función o actividad, de manera eficiente, eficaz y creativa, conforme a la naturaleza del trabajo. Capacidad productiva de un individuo que se define y mide en términos de desempeño real y demostrando 
en determinado contexto de trabajo y que no resulta sólo de la instrucción, sino que, de la experiencia en situaciones concretas de ejercicio ocupacional" La Oficina Internacional del Trabajo.

El concepto de competencia básica tiene que ver con la capacidad de los estudiantes para extrapolar lo que han aprendido y aplicar sus conocimientos ante nuevas circunstancias, su relevancia para el aprendizaje a lo largo de la vida y su regularidad" (OCDE, 2005).

Conjunto de conocimientos, habilidades y destrezas, tanto específicas como transversales, que debe reunir un titulado para satisfacer plenamente las exigencias de los contextos sociales".

Las definiciones de competencias presentadas evidencian la diversidad de conceptualizaciones existentes de ellas, desde visiones elementales de tipo conductistas hasta aquellas avanzadas de tipo holísticas. Un análisis de la noción de competencias entrega las siguientes cualidades de ellas (Vargas, F., 2001).

- Son características permanentes de las personas.

- Se ponen de manifiesto cuando se ejecuta una tarea o un trabajo.

- Están relacionadas con la ejecución exitosa de una actividad.

- Tienen una relación causal con el rendimiento laboral, es decir, no están asociadas con el éxito sino que se asume que realmente lo causan.

- Pueden ser generalizadas a más de una actividad.

- Combinan lo cognoscitivo, lo afectivo, lo conductual.

\section{Categorías de competencias}

Se han propuesto también numerosas clasificaciones de las competencias, dependiendo del contexto en el cual se utilizan y de las comprensiones e intereses de los autores. La siguiente es una lista no exhaustiva de aquellas comúnmente encontradas en la literatura.

- Saber, Saber hacer, Saber ser.

- Básicas, Genéricas, Específicas.

- Básicas, Genéricas, Laborales de Gestión, Laborales Técnicas Específicas.

- Duras, Blandas.

- Técnica, Metodológica, Social, Participativa. 
- Cognitivas, Procedimentales, Actitudinales.

- Conocimientos, Habilidades, Actitudes, Valores.

- Transversales, Específicas de la Profesión, Específicas del Actuar Profesional, de Gestión.

- Intrapersonales, interpersonales, profesionales y disciplinares.

- Conceptuales, Metodológicas, Humanas, de Alta Dirección.

Es necesario notar que las competencias identificadas para un perfil profesional pueden ser agrupadas siguiendo diferentes categorías. Así por ejemplo, las mismas competencias clasificadas como cognitivas, procedimientales y actitudinales, pueden ser clasificadas como básicas, genéricas y específicas, o como intrapersonales, interpersonales, profesionales y disciplinares, o duras y blandas, etcétera. También se podrían combinar categorías, como "conocimientos duros" y "conocimientos blandos", o "cognitivas transversales", entre otros. La elección de una taxonomía determinada, será decisión de quienes utilizarán la noción de competencias en un contexto determinado (laboral, educativo, de investigación, etcétera). Su elección conduce a opciones particulares para su desarrollo y evaluación.

\section{Necesidad de cambios en Educación Superior}

Dado el escenario social y tecnológico en permanente cambio que caracteriza el mundo moderno, donde el conocimiento evoluciona rápidamente, así como también los problemas y desafíos que la humanidad debe enfrentar y resolver ¿cuál es el rol que le corresponde a los sistemas educativos, en particular a la Educación Superior?

\section{Rol de la Educación Superior}

Estudios sobre el rol de la educación en el siglo XXI la sitúan como el instrumento fundamental para preparar las próximas generaciones de hombres y mujeres, con capacidad para dar solución a los numerosos desafíos que la humanidad enfrentará. En esta misión, la Educación Superior es la llamada a proveer a la sociedad del capital humano avanzado. Este rol trascendente de las universidades ha sido expresado en diversos contextos. 
La UNESCO, en la sección Pistas y Recomendaciones del informe de la Comisión Internacional sobre la educación para el siglo XXI, encargado por la UNESCO, menciona las siguientes cuatro "funciones esenciales" para las Universidades (Delors, 1996):

1. La preparación para la investigación y para la enseñanza;

- Como lugar de ciencia y fuente de conocimiento que llevan a la investigación teórica o aplicada, o a la formación de profesores;

2. La oferta de tipos de formación muy especializados y adaptados a las necesidades de la vida económica y social;

- Como medio de adquirir calificaciones profesionales conforme a unos estudios universitarios y unos contenidos adaptados constantemente a las necesidades de la economía, en los que se aúnen los conocimientos teóricos y prácticos a un alto nivel;

3. La apertura a todos para responder a los múltiples aspectos de lo que llamamos educación permanente en el sentido lato del término;

- Como plataforma privilegiada de la educación durante toda la vida, al abrir sus puertas a los adultos que quieran reanudar los estudios, adaptar y enriquecer sus conocimientos o satisfacer sus ansias de aprender en todos los ámbitos de la vida cultural;

4. La cooperación internacional;

- Como interlocutor privilegiado en una cooperación internacional que permita el intercambio de profesores y estudiantes, y facilite la difusión de la mejor enseñanza mediante cátedras internacionales.

El informe también destaca el rol social de la Universidad como "poder intelectual de la sociedad que la ayude a reflexionar, comprender y actuar", así expresa:

La universidad debe asimismo poder pronunciarse con toda independencia y plana responsabilidad sobre los problemas éticos y sociales -como una especie de poder intelectual que la sociedad necesita para que la ayude a reflexionar, comprender y actuar. Además encontraría de nuevo el sentido de su misión intelectual y social en la sociedad, siendo en cierto modo una de las instituciones garantes de los valores universales y del patrimonio cultural. 
En otro contexto, en la celebración de los 900 años de la Universidad de Bolonia, 400 rectores de universidades europeas destacaron el rol de las universidades, declarando (Magna Charta Universitatum, 1988 citado en $<\mathrm{http}: / /$ www.magna-charta.org/pdf/mc_pdf/mc_spanish.pdf $>$ ):

1. Que el porvenir de la humanidad, al finalizar este milenio, depende en gran medida del desarrollo cultural, científico y técnico, que se forja en los centros de cultura, de conocimiento y de investigación en que se han convertido las auténticas universidades;

2. Que la tarea de difusión de los conocimientos que la universidad ha de asumir respecto a las nuevas generaciones, implica hoy, que se dirija también al conjunto de la sociedad cuyo porvenir cultural, social y económico exige especialmente un considerable esfuerzo de formación permanente;

3. Que la universidad debe asegurar a las futuras generaciones la educación y la formación necesarias que contribuyan al respeto de los grandes equilibrios del entorno natural y de la vida.

Las esperanzas puestas en la Educación y particularmente en la Educación Superior, como medio para formar el capital humano que se hará cargo de las necesidades de la sociedad en el futuro próximo, requiere de reformas en los sistemas educativos.

\section{Presiones en Educación Superior}

Diversos factores presionan por reformas a las instituciones de Educación Superior en todo el mundo. Estudios internacionales han identificado los siguientes (De Boer, et al.) y (Vincent-Lancrin, S., 2004):

- El desarrollo de las tecnologías de la información y de las comunicaciones.

- La lógica de mercado de la Educación Superior.

- La globalización, internacionalización y regionalización.

- El avance de las redes sociales y de economías.

- El avance de la sociedad o economía del conocimiento.

- Tendencias socio culturales.

- Tendencias demográficas.

- Gobierno y financiamiento; liberalización. 
- Diversidad de proveedores.

- Aprendizaje durante toda la vida.

Otros factores que presionan por reformas en Educación Superior derivan de iniciativas de gobierno en países que buscan adecuar la educación a las necesidades de avanzar hacia sociedades más desarrolladas:

- Políticas en Educación Superior, relativos al aseguramiento de la calidad, al financiamiento, y a la certificación de competencias.

- Iniciativas para facilitar el reconocimiento de titulaciones y la movilidad estudiantil entre países, tales como el proyecto Tuning América Latina, y el establecimiento de Sistemas de Créditos Transferibles.

- Aumento del acceso a la Educación Superior, especialmente de estudiantes con deficiencias en competencias académicas básicas, de niveles socioeconómicos desfavorecidos.

- Mercado de la Educación Superior altamente competitivo, con participación importante del sector privado nacional e internacional.

- Frente a estos nuevos escenarios, las instituciones de Educación Superior, en general, no han dado respuestas adecuadas, pues enfrentan también desafíos internos, entre los cuales son recurrentes:

- Baja capacidad de gestión universitaria y de liderazgo para impulsar y conducir procesos de mejoramiento del desempeño académico.

- Baja pertinencia curricular de las carreras, incluyendo perfiles de egreso, planes de estudios, actividades de enseñanza y de aprendizaje, gestión del proceso educativo, entre otros.

- Deficiente idoneidad de los profesores y encargados de la gestión docente para conducir y aplicar los cambios requeridos para la formación de competencias de los nuevos perfiles. Además, la situación se complica debido al aumento de la edad de los docentes.

\section{Deficiencias detectadas en la formación profesional}

A nivel de América Latina el resultado de los procesos formativos presenta deficiencias relevantes en la formación de competencias consideradas importantes. Los análisis de los estudios realizados en el marco del proyecto Tuning América Latina, revelaron que "la mayor parte de las competencias considera- 
das como importantes por los cuatro grupos presenta brechas significativas, al compararlas con su grado de realización".

Las competencias genéricas que resultaron con mayor importancia, fueron:

- Capacidad de aplicar los conocimientos en la práctica.

- Capacidad de aprender y actualizarse.

- Capacidad para identificar, plantear y resolver problemas.

- Compromiso ético.

- Compromiso con la calidad.

- Capacidad para tomar decisiones.

- Capacidad de análisis y síntesis.

- Trabajo en equipo.

Las competencias genéricas que resultaron valoradas como menos importantes, fueron:

- Capacidad de comunicación en un segundo idioma.

- Habilidad para trabajar en contextos internacionales.

- Compromiso con la preservación del medio ambiente.

- Compromiso con su medio sociocultural.

- Habilidades interpersonales.

- Valoración y el respeto por la diversidad y multiculturalidad.

A fin de interpretar correctamente estos resultados, es necesario notar que a los participantes se les pidió opinaran sobre el grado de importancia de las competencias "para el trabajo en su profesión". Sin duda que los resultados serían distintos si hubiesen tenido que expresar sus ideas, por ejemplo, sobre el grado de importancia de la competencia para "contribuir, desde su profesión, al desarrollo de una sociedad con mejor calidad de vida", o "más solidaria", o "más sustentable", etcétera. La orientación de lo pedido en la encuesta justificaría que hayan resultado como menos importantes, competencias tan relevantes para enfrentar los desafíos del siglo XXI, como la "valoración y respeto por la diversidad y multiculturalidad", o el "compromiso con la preservación del medio ambiente", o la "habilidad para trabajar en contextos internacionales". Las consecuencias para los procesos formativos, en un caso y en el otro, son muy diferentes.

En el campo disciplinar específico de la ingeniería, los resultados de un estudio sobre la percepción, la industria sobre los graduados de ingeniería mostró los siguientes puntos débiles: 
- Incomprensión de los procesos de negocio.

- Tendencia a soluciones complicadas y de alta tecnología.

- Falta de capacidad de diseño o creatividad.

- Falta de consideración de alternativas.

- Falta de apreciación por la variedad.

- Todos quieren ser analistas.

- Mala comprensión de los procesos en proyectos de ingeniería.

- Visión restringida de la ingeniería y disciplinas afines.

- Debilidad en capacidades de comunicación.

- Pocas habilidades o experiencia para trabajar con otros en equipo.

- Tendencia al trabajo individual.

Los antecedentes presentados respecto a los cambios que están generando nuevos contextos sociales y tecnológicos, las nuevas necesidades formativas de las personas y los resultados de la formación profesional, reflejan la necesidad de reformas en la educación superior para que ésta de respuesta actualizada y pertinente, para formar las competencias requeridas por los profesionales para el ejercicio competente en las sociedades modernas.

\section{Hacia una nueva educación orientada al desarrollo de competencias}

El enfoque de competencias en educación ha ido adquiriendo mayor aceptación e importancia tanto a niveles de políticas en educación como también en el mundo académico y productivo, siendo uno de los temas centrales de los debates sobre reformas de la educación superior para el siglo XXI. Respecto a las propuestas de articulación e integración de sistemas educativos que se están promoviendo en iniciativas a nivel internacional, el enfoque de competencias contribuye a:

- Desarrollar un nuevo paradigma en educación, primordialmente centrada en el estudiante y la necesidad de encauzarse hacia la gestión del conocimiento.

- Responder a las demandas crecientes de una sociedad de aprendizaje permanente y de una mayor flexibilidad en la organización del aprendizaje. 
- Contribuir a la búsqueda de mayores niveles de empleabilidad y ciudadanía.

- Propiciar un impulso para la construcción y consolidación del Espacio América Latina, el Caribe y la Unión Europea de Educación Superior.

- Estimular acuerdos para la definición de un lenguaje común, que facilite el intercambio y diálogo entre los diferentes grupos interesados.

\section{Aportes de la formación basada en competencias}

Frente a los desafíos que plantea el mundo moderno a las personas, la formación orientada al desarrollo de competencias proporciona beneficios, como los siguientes:

Para las instituciones de educación superior:

- Impulsa la constitución de una universidad que ayuda a aprender constantemente y también enseña a desaprender.

- Supone transparencia en la definición de los objetivos que se fijan para un determinado programa.

- Incorpora la pertinencia de los programas, como indicadores de calidad, y el diálogo con la sociedad.

Para los docentes:

- Propulsa trabajar en el perfeccionamiento pedagógico del cuerpo docente.

- Ayuda en la elaboración de los objetivos, contenidos y formas de evaluación de los planes de estudio de las materias, incorporando nuevos elementos.

- Permite un conocimiento y un seguimiento permanente del estudiante, para su mejor evaluación.

Para los estudiantes y graduados:

- Permite acceder a un currículo derivado del contexto, que tenga en cuenta sus necesidades e intereses y provisto de una mayor flexibilidad.

- Posibilita un desempeño autónomo, el obrar con fundamento, interpretar situaciones, resolver problemas, realizar acciones innovadoras.

- Implica la necesidad de desarrollar: el pensamiento lógico, la capacidad de investigar, el pensamiento estratégico, la comunicación verbal, el dominio de otros idiomas, la creatividad, la empatía y la conducta ética. 
- Contribuye a tomar preponderante el autoaprendizaje, el manejo de la comunicación y el lenguaje.

- Prepara para la solución de problemas del mundo laboral, en una sociedad en permanente transformación.

- Prioriza la capacidad de juzgar, que integra y supera la comprensión y el saber hacer.

- Incluye el estímulo de cualidades que no son específicas de una disciplina, o aún de características específicas a cada disciplina, que serán útiles en un contexto más general, como en el acceso al empleo y en el ejercicio de la ciudadanía responsable.

Para los empleadores:

Conjuga los ideales formativos de la universidad con las demandas reales de la sociedad y del sector productivo.

Proporciona graduados capacitados en el manejo de las nuevas tecnologías de la informática y la comunicación, con posibilidades para operar con creatividad en distintos campos, científico, técnico, económico, social y ético.

\section{Desafios en la formación de competencias}

Los grandes desafíos de la formación de competencias se refieren a la aplicación del enfoque de competencias en las tareas concretas de la enseñanza y el aprendizaje. Las siguientes preguntas de reflexión y debate sobre el potencial de las competencias, surgieron producto de la reflexión conjunta a nivel latinoamericano (Tuning America Latina Proyect en $<$ http://tuning.unideusto.org/ tuningal $/>)$ :

- ¿Qué significa educar para el desarrollo de las competencias?

- ¿Cómo sería una enseñanza basada en competencias?

- ¿Cuáles serían los modelos pedagógicos más adecuados?

- ¿Cómo se construye un currículo basado en competencias?

- ¿Cómo se lleva a la práctica educativa el desarrollo de las competencias?

- ¿De qué modo se podría evaluar por competencias?

- ¿Cómo incorporar nuevas competencias, que se relacionen con las necesidades que surgen? 
- ¿Cómo diseñar competencias que preparen a los estudiantes para anticiparse a los cambios que se avecinan?

- ¿De qué manera se integra la generalidad de las competencias a las particularidades institucionales y nacionales?

- ¿De qué manera se pueden incluir, en el currículum de formación de profesores, competencias que permitan fortalecer la interculturalidad presente en el contexto latinoamericano?

A nivel de la formación profesional el entusiasmo de los últimos años en relación al tema de la formación por competencias ha movilizado muchos recursos y personas, no observándose todavía respuestas estables y maduras que sirvan de referencias confiables para la elaboración de propuestas educativas para el desarrollo de competencias. En todo caso, esto no debiera ser un objetivo final, pues las respuestas a estas preguntas deben considerar los contextos socioculturales y económicos de cada grupo humano, lo cual, en los países de América Latina al menos, tiene una gran diversidad.

\section{Currículum orientado al desarrollo de competencias}

La concepción del currículum ha tenido distintas orientaciones a través de los años (Gimeno, s/f) cada tipo de concepción curricular conduce a un tipo de praxis curricular con consecuencias diferentes para profesores, estudiantes y la sociedad.

Un currículum para el desarrollo de competencias deberá definir actividades educativas, modalidades de enseñanza y de aprendizaje, los medios recursos materiales y humanos, y los mecanismos de evaluación, organizados de tal forma que favorezcan el desarrollo de conocimientos, habilidades, actitudes, valores, etc., en escenarios adecuados para que los estudiantes adquieran las competencias declaradas en el perfil de egreso.

Los elementos curriculares más relevantes que deben ser reconsiderados para una formación orientada a la formación de competencias, son: el perfil de egreso, los planes de estudios, las actividades curriculares, las estrategias de enseñanza, de aprendizaje y de evaluación, los recursos didácticos, la gestión de los aprendizajes de los estudiantes. 


\section{El perfil de egreso}

El perfil de egreso establece las competencias profesionales que habrán desarrollado los estudiantes al egresar de una carrera. Como tal corresponde al resultado de un proceso formativo y representa el aporte que una institución de educación hace a la sociedad y a las personas. Ellos son la referencia principal para el diseño y construcción de los planes de estudios, programas de asignaturas, prácticas docentes, evaluación de aprendizajes, recursos didácticos, etcétera. Éstos se diseñan, construyen y ejecutan con el fin principal de lograr que los estudiantes desarrollen el perfil de egreso establecido en sus carreras.

A continuación se presenta un grupo de competencias agrupadas bajo las dimensiones profesional, personal y social, a modo de establecer algunos elementos que deberán visualizarse en la formación de profesionales.

\section{Dimensión profesional}

Se refieren a las cualidades transversales de tipo profesional que deberán expresar las personas en el ejercicio de su profesión.

- Actuar profesional fundamentado en el conocimiento científico y técnico disciplinar, con orientación de servicio al cliente y responsabilidad social, y con apego a la ética profesional.

- Actualización permanente en temas relativos a las ciencias, técnicas y tecnologías que fundamentan, apoyan y contribuyen a la disciplina, mejorando el conocimiento de sus avances y de su aplicación.

- Comprensión amplia del acontecer social, nacional y global, en los aspectos humanos, políticos, económicos, tecnológicos y medioambientales, en particular aquel que se relaciona con la disciplina.

- Manejo avanzado de las tecnologías de la información y de las comunicaciones, búsqueda, selección y análisis de información en web, navegación segura y conducta ética en internet.

- Trabajo eficaz con otras personas, en equipos multidisciplinarios y multiculturales, tanto en contextos nacionales como internacionales, en forma presencial o a distancia. 
Estas competencias se sustentan en el desarrollo y movilización articulada de un conjunto de conocimientos, habilidades y actitudes que incluyen, sin ser una lista exhaustiva, los siguientes: el conocimiento disciplinar, la comunicación oral, escrita y multimedial, el compromiso con valores universales, la responsabilidad social, la autonomía en el aprender, la búsqueda del crecimiento personal y social, el manejo eficiente de TICs, la capacidad de análisis y de síntesis, la comunicación en lengua extranjera, particularmente el inglés, el pensamiento crítico, la comprensión de la diversidad cultural.

\section{Dimensión personal}

Además de las competencias esenciales de las personas en el mundo contemporáneo, como la capacidad de leer y escribir, y de comunicarse en forma oral, escrita y multimedial, se deben sumar a ellas las deseables para un profesional:

- Actitud de crecimiento personal permanente, que se concreta con el desarrollo del conocimiento y conciencia de su persona, la disciplina y organización personal, el cuidado de la salud física, mental y emocional, el interés por adquirir nuevos conocimientos, el manejo del stress, la auto motivación, la rescilencia, la auto superación, entre otras

- Capacidad de razonamiento reflexivo crítico autónomo, lo que requiere de la persona la práctica del razonamiento fundamentado en datos e información veraz, la búsqueda de mayores niveles de comprensión, la habilidad para buscar y tratar datos e información, la capacidad para analizar y sintetizar ideas, el desarrollo de una visión holística, la autonomía para formular juicios, entre otras

- Conocimiento y manejo de las nuevas TICs, en un nivel suficiente para comunicarse vía mensajes de correo y Chat electrónicos, redactar textos y crear presentaciones multimediales, y el uso de planillas electrónicas.

- Capacidad y disposición al emprendimiento, una competencia cada vez más importante frente a la inestabilidad del mundo laboral, que se sustenta en una actitud de desarrollo personal, la iniciativa y perseverancia, la orientación al logro, la búsqueda de oportunidades, el manejo finan- 
ciero y la micro economía, la disposición a asumir riesgos, la rescilencia, entre otros.

\section{Dimensión social}

- Corresponden a las competencias que debiera expresar un profesional en su relación con el mundo social en el que participa y que se resumen en las siguientes:

- Interacción humana colaborativa, buscando generar relaciones sanas con otras personas, lo cual requiere el desarrollo de la comunicación oral, escrita y corporal, el respeto por las ideas ajenas, el manejo del diálogo, la práctica de buenas costumbres de comportamiento social, la conciencia del propio actuar y de su efecto en otras personas, el manejo de conflictos, entre otros.

- Relación constructiva con el mundo social del cual participa, actuando proactivamente en las organizaciones en que participa, respetando y valorando la diversidad humana y cultural, y destacando la responsabilidad social, particularmente el cuidado del medio ambiente.

- Compromiso y respeto de valores éticos universales, entre los que destacan el apego a la verdad y a la justicia, la defensa de los derechos humanos, el respeto a la legislación vigente, el reconocimiento de la diversidad cultural.

- Solidaridad, especialmente con los más desvalidos de la sociedad.

\section{Los planes de estudios}

Los planes de estudios son organizaciones de experiencias de enseñanza y de aprendizaje propuestas para una carrera, cuyo objetivo principal es dar respuesta a las necesidades formativas que derivan del perfil de egreso. Entre ellas se encuentran una variedad amplia de opciones tales como clases, talleres, desarrollo de proyectos, investigación, visitas a empresas, pasantías, entre otras. Éstas deben estar convenientemente concebidas, diseñadas, planificadas y ejecutadas para un óptimo avance y logro de los aprendizajes esperados en los estudiantes. 
Para la formación de competencias, los planes de estudios deben incluir actividades integradoras de aprendizajes en contextos que evoquen los escenarios en que las competencias deberán expresarse. De esta manera, los estudiantes podrán desarrollar y movilizar de manera articulada los elementos cognitivos, procedimentales y actitudinales asociados al "ser competente" en su futura profesión. Algunas cualidades que debieran incluir los nuevos planes de estudios, son:

a) Orientación al desarrollo de las competencias del perfil de egreso

- La formación se orienta claramente a formar las competencias del perfil de egreso, a través de resultados de los aprendizajes.

b) Flexibilidad

- Para permitir variantes formativas, el tratamiento de temas nuevos y el ajuste de los estudios al interés, talento y decisión de cada estudiante.

c) Contacto temprano del estudiante con su especialidad

- Para facilitar el desarrollo de su identidad profesional, mejorar la motivación y la satisfacción con sus estudios.

d) Orientación al aprender haciendo

- Aprendizaje activo colaborativo para favorecer el desarrollo de niveles avanzados de aprendizajes, y de competencias profesionales transversales.

e) Actividades integradoras de saberes

- Actividades para integrar conocimientos, en escenarios que evoquen y emulen los contextos de ejercicio profesional, en vinculación con el medio social y productivo.

Los planes de estudio debieran considerar, cuando sea pertinente, otras cualidades para satisfacer demandas de los sistemas de educación superior nacionales o internacionales, tales como criterios para la acreditación de carreras y mecanismos de articulación con otros niveles de formación.

\section{Actividades curriculares}

Las actividades curriculares deberán concebirse y configurarse de tal forma que establezcan claramente los elementos de competencia que se desarrollan y fortalecen con cada actividad. Deberá comunicar la modalidad de trabajo de los estudiantes, los términos para realizarla, los productos esperados, los calenda- 
rios, los recursos, las evaluaciones, etcétera. En ellas se deben especificar los resultados de los aprendizajes, como acciones observables y medibles que los estudiantes serán capaces de realizar después de haber realizado con éxito la actividad curricular. Estos son la referencia principal para el trabajo que estudiantes y profesores realizarán durante el desarrollo de la actividad curricular, y para las estrategias de evaluación.

Para cada actividad curricular se deben analizar y dar respuestas a las siguientes interrogantes:

- ¿A qué elemento(s) del perfil de egreso contribuye la asignatura?

- ¿Qué aprendizajes específicos se espera que logren los estudiantes en la asignatura?

- ¿Qué serán capaces de hacer los estudiantes al aprobar la asignatura?

- ¿Qué actividades realizarán los estudiantes para lograr los aprendizajes esperados?

- ¿Cómo apoyará/conducirá el profesor el proceso de aprendizaje de sus estudiantes?

- ¿Cómo evidenciarán los estudiantes lo que han aprendido?

- ¿Cómo verificará el profesor el progreso y logro de los aprendizajes de sus estudiantes?

\section{Docencia para el desarrollo de competencias}

En general, la educación superior ha centrado su enseñanza en la idea de la "transferencia de conocimiento" desde quienes lo poseen y actúan como fuente de él hacia quienes deben adquirirlo y que actúan como sus depositarios. Esta práctica sitúa al profesor al centro del proceso de enseñanza y de aprendizaje, pues es éste quien hace el esfuerzo principal para "transferir" el conocimiento que posee. Los estudiantes por su parte, a partir de las explicaciones de los profesores, y de sus estudios personales y grupales, deben "adquirir" el conocimiento y demostrar su apropiación. Esta concepción de la enseñanza y del aprendizaje es aún dominante en muchas instituciones de educación superior. Ella no favorece la formación de niveles superiores del ámbito cognitivo, tales como el análisis, síntesis, evaluación, ni tampoco el desarrollo de competencias del perfil profesional tales como el aprender en forma autónoma, el trabajo en 
equipos multidisciplinarios, la capacidad de identificar, plantear y resolver problemas, la creatividad, etcétera.

La recomendación general para una docencia orientada al desarrollo de competencias, son las prácticas de diferentes modalidades didácticas que favorezcan el "aprendizaje activo". Este se da cuando los estudiantes, generalmente trabajando con otros, se involucran en la adquisición de conocimientos y habilidades, que les permitan alcanzar ciertos objetivos. En el aprendizaje basado en problemas (Barrows H.S. \& R. M. Tamblyn, 1980: 1), el problema propuesto a los estudiantes es el elemento motivador que, apoyados en la gestión que hacen los profesores, impulsa a los estudiantes a aprender las materias vinculadas al problema y aplicarlas para resolverlo. En este proceso, los estudiantes desarrollan también capacidades vinculadas a las competencias del perfil, tales como el aprendizaje autónomo, la capacidad para identificar, formular y resolver problemas, la capacidad de análisis, el trabajo con otros, etcétera.

Otra modalidad que favorece el desarrollo de competencias es el aprendizaje basado en proyectos (Thomas, John, 2000 citado en $<$ http://www.bobpearlman.org/BestPractices/PBL_Research.pdf $>$ ). Éstos generan los contextos de aprendizaje donde los estudiantes deben aplicar conocimientos adquiridos, o aprender otros nuevos, e integrarlos con otros de especialidades diferentes para alcanzar los objetivos del proyecto. Éstos permiten vincular el esfuerzo de los estudiantes a resolver necesidades sociales y del sector productivo, y también se pueden orientar al desarrollo de la creatividad y del emprendimiento.

El aprendizaje basado en proyectos es un enfoque muy empleado y tiene una diversidad grande de formas de realización. Una síntesis de sus principales cualidades es la siguiente (ibíd.):

- Es una estrategia central del currículum.

- Tiene larga duración (varias semanas, un semestre, un año).

- Es interdisciplinario.

- Tiene situaciones desafiantes y constructivas.

- Es centrado en los estudiantes, basado en aprendizaje colaborativo.

- Se vincula a problemas reales.

- Tiene resultados concretos.

- Tiene impactos en habilidades personales, grupales y de solución de problemas. 
El aprendizaje basado en problemas ha sido desarrollado y aplicado con éxito en ámbitos de formación de médicos, abogados e ingenieros. Por otro lado, el aprendizaje basado en proyectos es altamente recomendado para la formación en ingeniería.

Un desafío importante en la docencia para el desarrollo de competencias, son las capacidades que deben poseer los profesores para aplicar las estrategias adecuadas, pues la tendencia dominante es la disposición para la "transmisión del conocimiento". La formación de competencias pasa necesariamente por un cambio de paradigma en la docencia, desde la idea de un profesor "proveedor" de conocimiento hacia una de "facilitador" del desarrollo de competencias de los estudiantes.

\section{Evaluación de competencias}

En general la evaluación siempre ha sido un tema controversial en educación. Aun cuando ésta se oriente a la evaluación de conocimientos adquiridos, los resultados de pruebas y exámenes no necesariamente reflejan el conocimiento logrado por los estudiantes. Son muchos los factores que influyen cuando se trata de evaluar. Por ejemplo, las disposiciones anímicas y corporales de los estudiantes no son neutrales durante un examen escrito u oral, lo que influye en sus capacidades de análisis y de síntesis. Por su parte, la evaluación de competencias es un tema con complejidad mayor, pues éstas se asocian a un "saber actuar en contexto". ¿Cómo evaluar en contexto competencias tales como "capacidad de aplicar creativamente el conocimiento disciplinar", o la "capacidad para funcionar en equipos multidisciplinares"?

La evaluación de competencias responde a criterios e indicadores establecidos para situaciones o familia de situaciones específicas en las cuales se desempeña un profesional. Esto hace de la evaluación de competencias un tema difícil de objetivar en términos cuantitativos, pues para demostrar la posesión de una competencia no basta con dar una prueba y obtener una nota. Estas no se desarrollan tomando un "curso de la competencia X" sino que se forman gradualmente mediante las actividades de aprendizaje que realizan los estudiantes. La formación de competencias requiere de un enfoque especial para evaluar los avances de los estudiantes hacia los niveles satisfactorios del "ser competente", 
el cual tendrá que evaluarse en muchos momentos del proceso. Técnicas asociadas a investigación y evaluación cualitativas, tales como portafolio de evidencias, focus groups, observación participante, auto y coevaluación, deberán ser consideradas.

La evaluación de competencias es un proceso de recolección de datos a partir de fuentes variadas que, considerando las experiencias de aprendizaje, permiten dar cuenta de:

- El nivel de desarrollo de las competencias del estudiante.

- Su grado de dominio de los recursos internos y externos que se movilizan en las competencias.

- El campo de acción donde el estudiante puede aplicar las competencias y los recursos.

La naturaleza compleja de la evaluación de competencias se refleja en la siguiente afirmación: "La evaluación de competencias debería incluir un insumo multivariado/multidimensional y multimétodo, incluyendo perspectivas múltiples en todos los niveles de desarrollo profesional" (Christiansen, M. D. et al., 2004). Asimismo, deben ser consideradas algunas cualidades que se identifican respecto a las competencias, bajo la concepción de "un saber actuar complejo". Estas son (Tardif, J.):

- Carácter integrador: cada competencia demanda el uso de una variedad de recursos.

- Carácter combinatorio: cada competencia se apoya en una combinación particular de recursos.

- Desarrollo incremental: cada competencia se desarrolla a lo largo de la vida.

- Carácter contextual: cada competencia se lleva a cabo en contextos que orientan la acción.

- Carácter evolutivo: cada competencia se concibe a fin de integrar nuevos recursos y nuevas situaciones sin que su naturaleza se comprometa.

En el enfoque de competencias la evaluación no está separada del proceso de aprendizaje, como lo es en el caso tradicional de una prueba o examen, que se toma después del aprendizaje. Para cada competencia a evaluar, se deben definir las conductas manifestadas que corresponden a diferentes niveles del logro de la competencia por parte del evaluado. También se deben definir indicadores 
que dan cuenta de la competencia, y las estrategias para obtener evidencias para dichos indicadores. Finalmente son necesarios criterios que, a partir de las evidencias colectadas, determinen el nivel de logro de la competencia.

\section{Conclusiones}

Incluso cuando existen importantes iniciativas de alcance nacional e internacional a nivel de gobiernos, instituciones y personas, para impulsar reformas en los sistemas de educación y orientar la formación de profesionales hacia el desarrollo de competencias, todavía persisten dificultades importantes para que este enfoque se instaure en la educación superior. Estas dificultades se sitúan en diversos ámbitos de los contextos educativos. Desde la necesidad por una mejor comprensión de los nuevos escenarios socioculturales, económicos, científicos y tecnológicos del mundo moderno, y de sus consecuencias sobre los nuevos perfiles profesionales, por parte de los responsables de gestionar los currícula, hasta las capacidades requeridas por profesores y profesoras para desarrollar y aplicar estrategias de enseñanza, de aprendizaje y evaluativas orientadas al desarrollo de competencias. En muchas instituciones de educación superior, las capacidades de gestión de sus directivos son insuficientes para impulsar cambios, especialmente en instituciones tradicionales dependientes del estado. Por otro lado, la resistencia del cuerpo académico a los cambios es también una dificultad mayor, especialmente cuando el peso de la tradición es fuerte y la edad avanzada. Las dificultades se acentúan cuando las políticas impulsadas por los gobiernos en temas de financiamiento y de aseguramiento de la calidad de la educación apuntan en direcciones diferentes, y cuando los incentivos a las universidades fomentan orientar el interés y esfuerzo de los profesores hacia actividades académicas más rentables, distintas a la tarea educativa. En estos escenarios las demandas por nuevas propuestas curriculares que respondan a las necesidades de formar las competencias que requieren los profesionales para el nuevo siglo, son postergadas o tratadas con poco interés.

Con muchas dificultades por superar, la formación orientada al desarrollo de competencias, constituye un gran desafío que las instituciones de educación superior y los gobiernos, no pueden postergar. La solución a los problemas complejos, inéditos y abiertos que enfrenta la humanidad requiere de profesio- 
nales con nuevas competencias, y las universidades están llamadas a proveer del contexto educativo que las haga posibles.

\section{Referencias bibliográficas}

\section{Textos}

Barrows H.S. \& R.M. Tamblyn. Problem-Based Learning: An Approach to Medical Education. New York: Springer Publishing Company. 1980.

Delors, J. La educación encierra un tesoro. Madrid: Ediciones UNESCO. 1996.

De Boer, Harry et al., Academia in the 21st Century: an analysis of trends and perspectives in higher education and research, AWT Achtergrondstudies N. . 28. Den Haag.

Christiansen, M. D. et al., Assessment of Competence. The Ultimate Frontier for Improving Professional Competence, 2004.

Gallart, M. A. y C. Jacinto. Competencias laborales: tema clave en la articulación educación-trabajo. Buenos Aires: CIID-CENEP.

Gimeno S. J. El currículum: una reflexión sobre la práctica. Madrid: Ed. Morata S.A. s/f.

Irigoin, M. y F. Vargas. Competencia laboral. Manual de conceptos, métodos y aplicaciones en el sector Salud. Montevideo: Cinterfor. 2000.

Le Boterf, G. Ingeniería de las competencias. Barcelona: Gestión 2000. 2001.

OCDE 2005. Informe PISA 2003. Aprender para el mundo de mañana. Madrid: Santillana.

McClelland, David. Testing for competence rather than for "intelligence". American Psychologist 28: 1-14. 1973.

Perrenoud. Competencias en la Formación y Competencias en la Gestión del Talento Humano. Convergencias y Desafíos. 2002.

Pinto, L. "Currículo por competencias. Necesidad de una nueva escuela". RevistaTarea, 43. s/f.

Rychen y Hersh. Definición y Selección de las Competencias (DeSeCo): Fundamentos teóricos y conceptuales de las competencias. OCDE, París. 2002. 
Spencer, L.M. y S.M. Spencer. Competence at Work. New York: John Wiley and Sons. $\mathrm{s} / \mathrm{f}$.

Tardif, J. L'évaluation des competences. Montreal, Canadá: Les Éditions des Chenelière Inc. $\mathrm{s} / \mathrm{f}$.

Tejada, J. "Acerca de las competencias profesionales". Revista Herramientas, 57. $\mathrm{s} / \mathrm{f}$.

Todd, R. H. Designing a Senior Capstone Course to Satisfy Industrial Customers. Journal of Engineering Education, April, 1993, p. 92-100.

Vargas, F. "Las reglas cambiantes de la competitividad global en el nuevo milenio. Las competencias en el nuevo paradigma de la globalización" en Revista Iberoamericana de Educación. OEI. 2001.

Vincent-Lancrin, S., Internationalisation and trade in higher education: opportunities and challenges. OCDE. 2004.

\section{Sitios web}

Competencia Laboral, Cinterfor, Centro Interamericano para el Desarrollo del Conocimiento en la Formación Profesional, <http:/www.ilo.org/public/ spanish/region/ampro/cinterfor/temas/complab/doc/otros/sel_efe/i.htm>

Ley de Sistema Nacional de Certificación de Competencias Laborales, Chile $<$ http://www.bcn.cl/leyes/272829>

$<$ http://www.capa.ca/Main certification-fr.html>

The National Skills Standards Board, <http://www.nssb.org/>

The Learning and Skills Council of UK, <http://www.lsc.gov.uk/>

Plan Nacional de Calificación, Ministerio de Trabajo y Empleo, Brasil, $<$ http:// www-ilo-mirror.cornell.edu/public/spanish/region/ampro/cinterfor/ifp/ sefor/pnq.pdf>

Tuning Educational Structures in Europe, <http://www.relint.deusto. es/>TUNINGProject/spanish/doc_fase1/Tuning Educational.pdf $>$

Tuning America Latina Proyect, <http://tuning.unideusto.org/tuningal/>

\section{Real Decreto 1513/2006, Ministerio de Educación y Ciencia, España,} $<$ http://noticias.juridicas.com/base_datos/Admin/rd1513-2006.html $>$ 
Programa de Mejoramiento de la Calidad de la Educación Superior, Ministerio de Educación, Chile, $<$ http://www.mecesup.cl $>$

Programa de Modernização e Qualificação do Ensino Superior, <http://portal. $\mathrm{mec}$.gov.br/sesu/index.php?option=content\&task=view \&id=439\&Item $\mathrm{id}=303>$

Modelos Educativos del Instituto Tecnológico de Estudios Superiores de Monterrey, México, <http://www.itesm.edu/wps/portal?WCM_GLOBAL_ CONTEXT=/wps/wcm/connect/ITESMv2/Tecnológico+de+Monterrey/ Conócenos/Modelo+educativo/>

Modelo Educativo de la Universidad de Deusto, España, <http://studieren.es/ uploads/tx_assearchengine/deusto.pdf $>$

Lohmann, J., Global Engineering Excellence: The Role of Educational R\&D, 36th Annual Brazilian Congress of Education in Engineering, Sao Paulo, Brazil, September 12, 2008. <http://www.provost.gatech.edu/subjects/ lohmann/resources/COBENGE 200812 Sep 08.pdf>

Educating Engineers for the $21^{\text {st }}$ Century, International Electron Devices Meeting, San Francisco, USA, 2008, <http://www.eng.ufl.edu/newsroom/ articles/detail_articles.php?id=993>

Graham, S. A. et al., Developing Methodologies to Integrate Content and Behavioral Skills: A Pragmatic Approach to Curriculum Design. Community/ Junior College Quarterly of Research and Practice 15/3 (1991): 285-293., citado en $<$ http://www.nwrel.org/scpd/sirs/8/c015.html>

Magna Charta Universitatum, Bolonia, 1988, <http:/www.magna-charta.org/ pdf/mc_pdf/mc_spanish.pdf $>$

Benoite, P. et al., Informe Final - Proyecto Tuning - América Latina, 2007, Universidad de Deusto, Universidad de Groningen, Bilbao, España, $<$ http:// tuning.unideusto.org>

Thomas, John, A Review of Research on Project-Based Learning, The Autodesk Foundation, 2000, <http://www.bobpearlman.org/BestPractices/ PBL_Research.pdf>

Envío 29 de abril/2011 - Aceptación 17 de noviembre/2011. 